\title{
PENALIZED LIKELIHOOD EMISSION IMAGE RECONSTRUCTION WITH UNCERTAIN BOUNDARY INFORMATION
}

\author{
Stephen R. Titus, Alfred O. Hero III, Jeffrey A. Fessler \\ 4401 EECS, University of Michigan, Ann Arbor, MI 48109
}

\begin{abstract}
In this paper, a method is introduced for incorporating perfectly registered MRI boundary information into a penalized likelihood emission reconstruction scheme. The boundary curve is modeled as a periodic spline whose coefficients are estimated from the MRI image. The resulting boundary estimate is mapped to a spatially variant set of Gibbs weights. When incorporated into a quadratic roughness penalty, these weights improve emission reconstruction bias/variance performance by preventing smoothing across the estimated boundary. Finally, we derive a new penalty function that accounts for the uncertainty inherent in the boundary estimates.
\end{abstract}

\section{INTRODUCTION}

Emission computed tomography (ECT) images of radioisotope concentration are reconstructed from data that consists of counts of detected decay events. Several factors, including dose limitations, scatter, attenuation, and detector efficiency, limit the acquired count rates achievable in ECT. The resulting low SNR gives rise to a poor bias/variance tradeoff. Recently, various researchers have used MRI boundary side information to improve performance in the case where functional and anatomical boundaries are coincident, e.g. [1],[2]. However, many use the side information "blindly", ignoring the uncertainty of the boundary estimate. Here, we present a spline-based method for incorporating an anatomical boundary estimate from an MRI image into the penalized likelihood (PL) emission reconstruction paradigm. Specifically, we focus on estimating mean uptake in a region of interest (ROI) inside the boundary curve. Estimator performance is characterized by the bias/variance tradeoff associated with varying the regularization parameter, and asymptotic limits on bias/variance performance are determined.

This work was supported by National Cancer Institute, ROICA-54362-02 and DOE Grant DE-FG02-87ER65061.

\section{MRI BOUNDARY ESTIMATION}

Consider an anatomical slice of interest which contains a single organ or tumor that selectively absorbs a relatively uniform concentration of radionuclide, so that the radionuclide and anatomical boundaries coincide. Let $r(x, y)$ denote the resulting single boundary curve present in the perfectly registered MRI and emission slice planes. The MRI boundary estimation problem consists of generating an estimate $\hat{r}(x, y)$ from $Y_{M}$, a noisy MRI image of the slice.

We parameterize boundary $r(x, y)$ using a periodic polar spline. A periodic polar spline function $r(\phi)$ of degree $d$ is a piecewise polynomial on $[0,2 \pi]$ that is defined over a set of $K$ scalar "knots" $\left\{x_{1}=0<x_{2}<\right.$ $\left.\ldots<x_{K}=2 \pi\right\}$, and has the following properties [3]: (i) it is a polynomial of degree $\leq d$ on intervals $\left[x_{j}, x_{j+1}\right]$, (ii) it is continuous and has $d-1$ continuous derivatives on $[0,2 \pi]$, and (iii) the values of the function and its first $d-1$ derivatives at 0 equal those at $2 \pi$. Any periodic polar spline function represents a smooth, closed polar curve with respect to a prescribed origin. The normalized b-spline basis for this set of splines (see [3]) allows us to write any periodic polar spline function $r(\phi)$ in terms of a set of $K-1$ basis splines:

$$
r_{\boldsymbol{\theta}}(\phi)=\sum_{i=1}^{K-1} \theta_{i} B_{i}(\phi) \quad \forall \phi \in[0,2 \pi] .
$$

The scalars $\theta=\left(\theta_{1}, \ldots, \theta_{K-1}\right)$ are called spline coefficients. Non-negativity of the $\left\{B_{i}(\cdot)\right\}$ assures that if all $\theta_{i}>0, r_{\theta}(\phi)$ represents a non-intersecting, continuous closed boundary.

Side information is extracted from the MRI image using ML estimation of $\theta$ [4]; i.e.

$$
\hat{\theta}=\operatorname{argmax}_{\boldsymbol{\theta}} \ln f\left(Y_{\boldsymbol{M}} ; \boldsymbol{\theta}\right) .
$$

We model the MRI scanner as a linear, spatially shift invariant system with a symmetric Gaussian 2D impulse response $h(x, y)$ and with additive white Gaussian noise [5]. These system and noise models lead to the following Gaussian model for MRI image $Y_{M}$ :

$$
Y_{M}=I(\theta) * * H+N
$$


where matrix $I(\theta)$ is the true MRI image containing boundary $\boldsymbol{\theta}, H$ contains samples of $h(x, y)$ at pixel centers, $N$ contains IID white noise, and operator " $* *$ " denotes $2 \mathrm{D}$ matrix convolution.

\section{PL RECONSTRUCTION}

The ECT reconstruction problem consists of estimating $n$ parameters $\lambda$, the radionuclide concentrations in the slice pixels, from a data set $\boldsymbol{Y}_{E}$ of detected counts on the surfaces of $m$ detectors. These counts are statistically independent and have Poisson statistics with rate parameters given by the elements of the vector $A \boldsymbol{\lambda}+\boldsymbol{r}$, where $A$ is an $m \times n$ matrix of rank $n$ corresponding to the system transition probabilities, and $r$ is a an offset in the detected rates due to a random background intensity level. In penalized likelihood (PL) reconstruction with side information, we maximize an objective that is the sum of the Poisson data log-likelihood and a penalty function $R(\boldsymbol{\lambda}, \hat{\boldsymbol{\theta}})$ :

$$
\begin{aligned}
\hat{\boldsymbol{\lambda}} & =\operatorname{argmax}_{\boldsymbol{\lambda}} J\left(\boldsymbol{Y}_{E} ; \boldsymbol{\lambda}\right) \\
J\left(\boldsymbol{Y}_{E} ; \boldsymbol{\lambda}\right) & =\left[\ln f\left(\boldsymbol{Y}_{E} ; \boldsymbol{\lambda}\right)-\beta R(\boldsymbol{\lambda}, \hat{\boldsymbol{\theta}})\right] .
\end{aligned}
$$

The term $R(\boldsymbol{\lambda}, \hat{\boldsymbol{\theta}})$ allows us to direct the reconstruction by "penalizing", via large $R$ values, the likelihood of images that are undesirable or are not in good agreement with prior information. It is often chosen proportional to some measure of roughness of $\lambda$, so that smoother (lower variance) images than those obtained via ML estimation are produced. The parameter $\beta$ in (4) establishes the bias/variance operating point (smoothness) by determining the relative importance of maximizing $\ln f\left(Y_{E} ; \lambda\right)$ versus minimizing the penalty term.

We select $R(\boldsymbol{\lambda}, \hat{\boldsymbol{\theta}})$ to implement a spatially variant quadratic roughness penalty, where smoothness is encouraged inside and outside, but not across, the boundary $\hat{\boldsymbol{\theta}}$ :

$$
R(\boldsymbol{\lambda} ; \hat{\boldsymbol{\theta}})=\sum_{k} \sum_{j} \omega_{k j}(\hat{\boldsymbol{\theta}})\left(\lambda_{k}-\lambda_{j}\right)^{2} .
$$

The summations above are over pixels, and $\left\{\omega_{k j}\right\}$ is a set of penalty weights that for fixed $k$ promote smoothing within the neighborhood of the $k$ th pixel. We use a Gibbs weighting scheme with a first order 2D pixel neighborhood consisting of four adjacent neighbors. By definition, the weights are symmetric $\left(\omega_{k j}=\omega_{j k}\right)$, and $\omega_{k j}$ can be non-zero only in the neighborhood of the $k$ th pixel (see [6]).

The side information is encoded into these weights as follows. Let $\boldsymbol{J}(\hat{\boldsymbol{\theta}})$ be an image whose $j$ th pixel inten- sity is equal to the fraction of pixel $j$ that falls inside curve $\hat{\boldsymbol{\theta}}$. Intrapixel weight $\omega_{j k}(\hat{\boldsymbol{\theta}})$ is

$$
\omega_{j k}(\hat{\boldsymbol{\theta}})=\left\{\begin{array}{clc}
1 & : & J_{j}(\hat{\boldsymbol{\theta}}) \geq .50, J_{k}(\hat{\boldsymbol{\theta}}) \geq .50 \\
1 & : & J_{j}(\hat{\boldsymbol{\theta}})<.50, J_{k}(\hat{\boldsymbol{\theta}})<.50 \\
0 & : & \text { else }
\end{array}\right\}
$$

Notice that only pixels more than half inside the boundary included in the interior.

The image that maximizes (4) can not be found analytically; therefore, an iterative method must be used. In the sequel we use the PML-SAGE3 algorithm of [7] to perform the maximization. This EM-type algorithm is characterized by monotonic, fast convergence.

\section{LARGE $\beta$ LIMIT}

Next we give the limiting form of the PL reconstruction error as $\beta$ gets large. In this limiting case the penalty $-\beta R(\boldsymbol{\lambda} ; \hat{\boldsymbol{\theta}})(5)$ dominates the objective (4) forcing $\lambda$ to be piecewise constant over the neighborhoods defined by Gibbs weights $w_{j k}(\hat{\theta})$. In particular, when the $w_{j k}$ 's correspond to Gibbs weights enforcing smoothness inside and outside of the estimated ROI, the limiting form of the PL objective function is

$$
J\left(\boldsymbol{Y}_{E}, \boldsymbol{\lambda}\right)=\left\{\begin{array}{ccl}
\ln f\left(\boldsymbol{Y}_{E} ; \boldsymbol{\lambda}\right), & \boldsymbol{\lambda}=S_{\hat{\boldsymbol{\theta}}^{\boldsymbol{\kappa}}} & \boldsymbol{\kappa} \in \mathbb{R}^{2} \\
-\infty, & \text { other } & \boldsymbol{\lambda}
\end{array}\right.
$$

where $S_{\hat{\theta}}=\left[\underline{1}_{R O I}, \underline{1}^{\prime}-\underline{1}_{R O I} \hat{\theta}_{\hat{\theta}}\right]$ is a matrix shose columns are indicator functions of the interior $\left(\underline{1}_{R O I}\right)$ and exterior $\left(1-\underline{1}_{R O I}\right)$ of the ROI, and $\kappa=\left[\kappa_{1}, \kappa_{2}\right]$ are constant levels within the interior and exterior, respectively.

In the high count regime the $\log$ likelihood $\ln f\left(\boldsymbol{Y}_{E} ; \boldsymbol{\lambda}\right)$ is well approximated (up to additive constants) by the Gaussian $\log$ likelihood $-\frac{1}{2}\left(\boldsymbol{Y}_{E}-A \boldsymbol{\lambda}\right)^{T} \Lambda^{-1}\left(\boldsymbol{Y}_{E}-A \boldsymbol{\lambda}\right)-$ $\frac{1}{2} \sum_{i} \ln [\Lambda]_{i i}$, where $\Lambda=\operatorname{diag}(A \lambda)$ is a diagonal matrix with the elements of the vector $A \lambda$ arranged along the diagonal, and $[\Lambda]_{i i}$ denotes the $i$-th diagonal element of the matrix $\Lambda$. Ignoring dependence of $\Lambda$ on the unknown $\boldsymbol{\lambda}$, the maximum of the PL objective function (7) is attained by the constrained linear least squares estimator $\hat{\lambda}^{c}=S \hat{\kappa}$ where

$$
\hat{\boldsymbol{\kappa}}=\left(S^{T} F_{\lambda} S\right)^{-1} S^{T} A^{T} \Lambda^{-1}\left(\boldsymbol{Y}_{E}-\boldsymbol{r}\right),
$$

and $F_{\lambda}=A^{T} \Lambda^{-1} A$ is the Fisher information matrix associated with the emission data, and we have suppressed the subscript $\hat{\boldsymbol{\theta}}$ from $S$ for simplicity.

Note that $\hat{\lambda}^{c}$ is an unimplementable ideal least squares estimator since it requires the matrix $\Lambda$ which depends on the unknown image $\boldsymbol{\lambda}$. However, the bias and variance performance of this ideal estimator will be used to bound the asymptotic performance of the estimator (4). The bias and the covariance of this estimator 
are $E\left[\hat{\lambda}^{c}\right]=\left[S\left(S^{T} F_{\lambda} S\right)^{-1} S^{T} F_{\lambda}-I\right] \lambda$ and $\operatorname{cov}\left(\hat{\lambda}^{c}\right)=$ $S\left(S^{T} F_{\lambda} S\right)^{-1} S^{T}$. When $\lambda=S \kappa$ the image $\lambda$ is truly piecewise constant over the estimated ROI and the bias is zero.

A useful approximation to $\hat{\lambda}^{c}$ can be implemented by replacing the unknown covariance $\Lambda=\operatorname{diag}\left(E\left[\boldsymbol{Y}_{E}\right]\right)$ in (8) by the empirical estimate $\operatorname{diag}\left(\boldsymbol{Y}_{E}\right)$. This can be used to initialize iterative optimization algorithms, e.g. grouped coordinate ascent, EM, or SAGE, for finding the true ML estimate $\hat{\lambda}$ of (4) when the smoothing parameter $\beta$ is large.

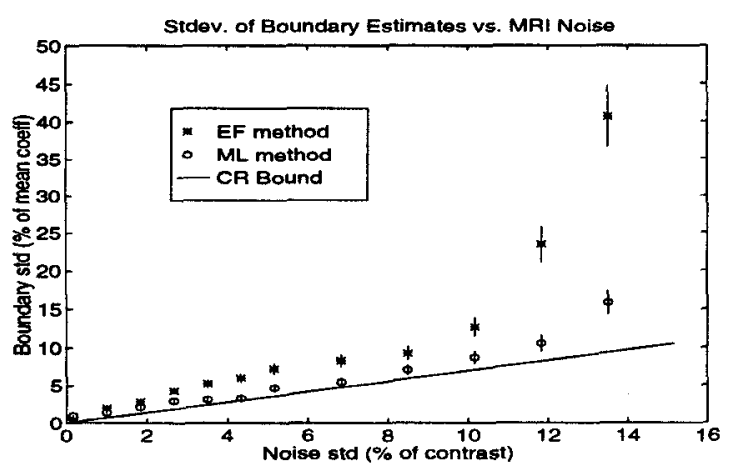

Figure 1: Variance of MRI boundary extraction methods as compared to the $\mathrm{CR}$ bound.

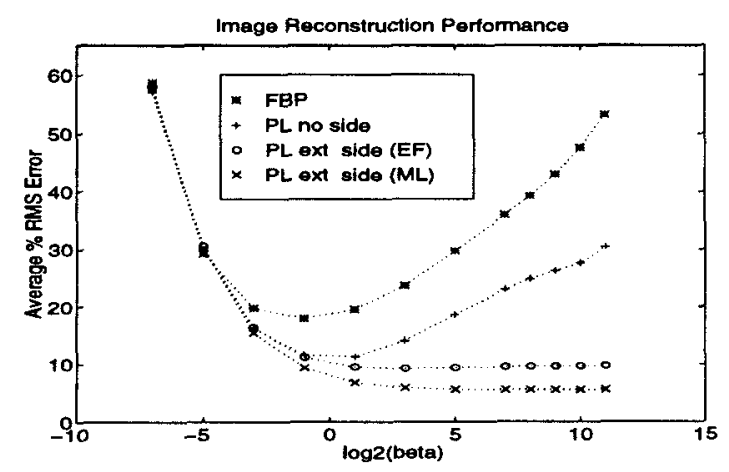

Figure 3: Average RMS reconstruction error for various emission reconstruction methods.

\section{RESULTS}

We generated 400 realizations of noisy MRI and ECT data to investigate the bias variance perfomance of ECT uptake estimation with and without MRI side information. The ROI boundary was a quadratic spline with $K=16$ equispaced knots which is a least squares fit to an ellipse with major axis 5 pixels and minor axis 3 pixels located at $(-10,0)$ pixels relative to image center. The contrast for both the MRI and ECT

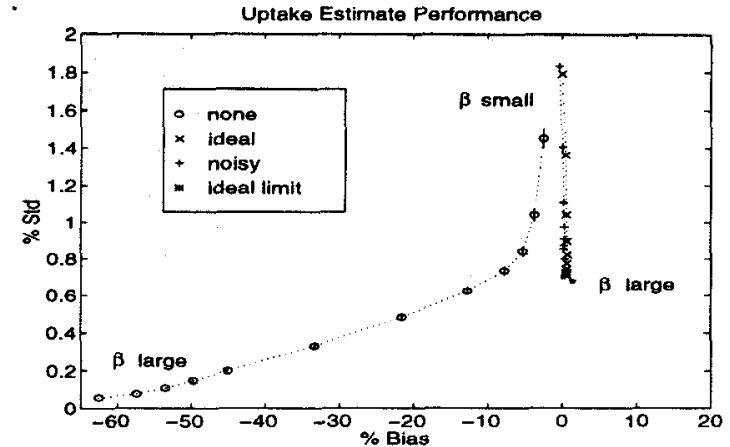

Figure 4: Comparison of empirical standard deviation vs. bias for no, ideal, and noisy (ML extracted) side information.

mean images were identical: for MRI the mean intensity inside the ROI was 6 in a zero mean background while for ECT the mean intensity inside the ROI was 9 in a background of mean 3. In both ECT and MRI images each boundary pixel was assigned mean intensity equal to the interior mean intensity times the fraction of the boundary pixel included inside the ROI. The MRI spatial blurring width was equal to $\sigma_{s}=0.75$ (approximately $19 \%$ of average ROI radius) and a spatially independent Gaussian noise was added having zero mean and standard deviation $\sigma_{n}=0.18$ (appoximately $3 \%$ of contrast). The ECT data was sampled by a parallel beam tomograph corresponding to PET projections over 64 radial bins, and 60 equispaced projection angles over $180^{\circ}$. Poisson noise was added to the ECT data by generating $10^{6}$ Poisson realizations of the mean intensity and adding $15 \%$ random coincidences.

In Fig. 1 we show the total standard deviation of the MRI boundary estimates, computed by summing estimation errors over a set equispaced angular samples of the boundary: std dev $=\sqrt{\sum_{i=1}^{N} \operatorname{var}\left(\hat{r}\left(\phi_{i}\right)\right)}$, based on both the maximum likelihood estimator (ML) and an edge filtering estimator (EF) discussed in [8]. Both estimators were observed to be virtually unbiased for noise standard deviation less than $10 \%$ of contrast. The EF boundary extractor is a computationally much simpler, but ad hoc, method as constrasted to ML which requires iterative methods (we used a quasi-Newton method) to solve the nonlinear least squares problem (2). It is significant that both boundary extraction methods virtually attain the $\mathrm{CR}$ bound on variance (derived in [8]) over a large range of noise power.

In Figures 2a-2d we show the emission phantom, the filtered back projection (FBP) reconstruction (FBP Hanning filter bandwidth chosen to match $\beta=8$ smooth- 
(a)

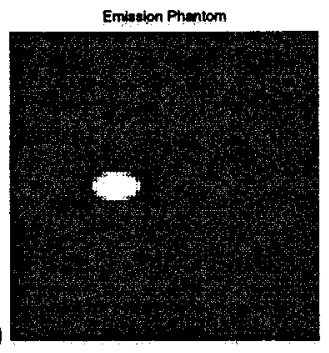

(b)

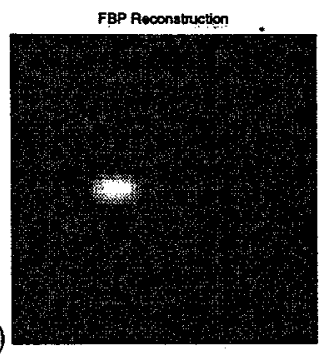

(c)

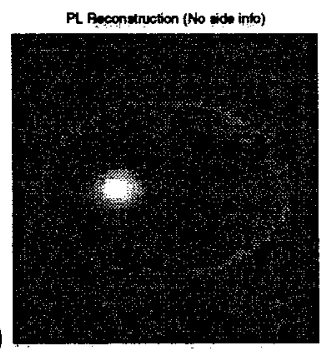

(d)

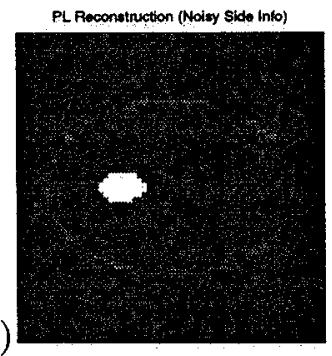

Figure 2: (a) Emission phantom. (b) FBP recon corresponding to $\beta=8$. (c) PL recon, $\beta=8$, no side information. (d) PL recon, $\beta=8$, noisy side information obtained by ML extraction.

ing of $\mathrm{PL})$, the PL reconstruction $(\beta=8)$ without MRI side information (all $w_{i j}$ set to 1 ), and PL reconstruction $(\beta=8)$ using $w_{i j}$ assignment based on extracted MRI boundaries using Maximum Likelihood estimation of $\hat{\boldsymbol{\theta}}$ in the spline model. All reconstructions in Figs. 2c and $2 \mathrm{~d}$ were performed using 60 iterations of the PMLSAGE3 algorithm initialized with the filtered back projection image. The qualitative difference between the severely blurred reconstructions (b) and (c) and the highly defined reconstruction (d) is obvious. Figure 3 shows quantitatively the improvement in terms of $\%$ RMS reconstruction error as a function of $\beta$ defined as

$$
\text { \%RMS error }=\frac{\sum_{i j}\left(\lambda_{i j}-\hat{\lambda}_{i j}\right)^{2}}{\sum_{i j} \lambda_{i j}^{2}} \times 100 .
$$

The large- $\beta$ divergence of the RMS error of FBP and PL without side information is due to excessive smoothing accross the boundary of the ROI.

Figure 4 shows empirical results for estimating the uptake inside boundary $\boldsymbol{\theta}$ as we vary the regularization parameter $\beta$. The uptake is defined as the sum of the total intensity within the ROI $\underline{1}_{R O I}^{T} \lambda$. Notice that the reconstructions using ideal side information suffer from essentially no bias, and approach the theoretical large $\beta$ limit of performance, labeled " + " in Fig. 4. This limit is computed as the coordinate pair $\left(\underline{1}_{R O I}^{T} E\left[\hat{\lambda}^{c}\right], \underline{1}_{R O I}^{T} \operatorname{cov}\left(\hat{\lambda}^{c}\right) \underline{1}_{R O I}\right)$ where the bias and covariance of the two-level constrained estimator $\hat{\lambda}^{c}$ were derived in the previous section. At all standard deviation levels, the bias using ideal side information is substantially less than without side information, with the performance gap increasing to more than a factor of 15 as the ideal performance limit is approached.

\section{CONCLUSIONS AND FUTURE WORK}

We have shown that under a spline boundary model for the MRI defined region of interest estimators of spline parameters can be effectively incorporated into ECT image reconstructions via penalized likelihood (PL) using an estimator-dependent Gibbs weighting scheme. This approach is blind to the fact that the MRI-based boundary estimator is noisy with approximately zero mean and variance equal to the inverse Fisher information matrix. A future paper will report on a technique which modifies the penalty function in the PL objective to discount the MRI spline estimator as a function of its variance.

\section{REFERENCES}

[1] G. Gindi, M. Lee, A. Rangarajan, and G. Zubal. Bayesian reconstruction of functional images using anatomical information as priors. IEEE Transactions on Medical Imaging, 12(4), December 1993.

[2] V. E. Johnson. A model for segmentation and analysis of noisy images. Journal of the American Statistical Association, 89(425):230-241, March 1994.

[3] J. H. Ahlberg. The Theory of Splines and Their Applications. Academic Press, 1967.

[4] Stephen R. Titus, Alfred 0. Hero, and Jeffrey A. Fessler. NMR object boundaries: B-spline modeling and estimator performance. In International Conference on Acoustics, Speech, and Signal Processing, 1995.

[5] W.A. Edelstein. Intrinsic signal to noise ratio in NMR imaging. Magnetic Resonance in Medicine, 1986.

[6] T. Hebert and R. Leahy. A generalized EM algorithm for 3-D Bayesian recon struction from Poisson data using Gibbs priors. IEEE Transactions on Medical Imaging, 8(2):194-202, June 1989.

[7] J. A. Fessler and A.O. Hero. Penalized maximum likelihood image reconstruction using space alternating generalized em algorithms. IEEE Transactions on Image Processing, 4(10), October 1995.

[8] Stephen R. Titus. Improved penalized likelihood reconstruction of anatomically correlated emission computed tomography data. PhD thesis, The University of Michigan, Ann Arbor, December 1996. 